\title{
Reciprocity as an Ever-Present Dual Property of Everything
}

\author{
Hans Hermann Otto \\ Department of Materials Science and Crystallography, Clausthal University of Technology, Clausthal-Zellerfeld, Germany \\ Email: hhermann.otto@web.de
}

How to cite this paper: Otto, H.H. (2020) Reciprocity as an Ever-Present Dual Property of Everything. Journal of Modern Physics, 11, 98-121.

https://doi.org/10.4236/jmp.2020.111007

Received: October 29, 2019

Accepted: January 11, 2020

Published: January 14, 2020

Copyright $\odot 2020$ by author(s) and Scientific Research Publishing Inc. This work is licensed under the Creative Commons Attribution International License (CC BY 4.0).

http://creativecommons.org/licenses/by/4.0/

\section{(c) (i) Open Access}

\begin{abstract}
Reciprocity may be understood as relation of action and reaction in the sense of Hegels philosophical definition. Quoting Kant, freedom and ethical necessities are reciprocally limited. In this contribution, a more mathematical than philosophical reflection about reciprocity as an ever-present dual property of everything was given. As a crystallographer, the author is familiar with the action of Fourier transforms and the relation between a crystal lattice and its reciprocal lattice, already pointing to the duality between particles and waves. A generalization of the reciprocity term was stimulated by results of the famous Information Relativity ( IR) theory of Suleiman with its proven physical manifestation of matter-wave duality, compared to the set-theoretical E-Infinity theory developed by El Naschie, where the zero set represents the pre-quantum particle, and the pre-quantum wave is assigned to the empty set boundary surrounding the pre-particle. Expectedly, the most irrational number $\varphi=(\sqrt{5}-1) / 2$ of the golden mean is involved in these thoughts, because this number is intimately connected with its inverse. An important role plays further Hardy's maximum quantum entanglement probability as the fifth power of $\varphi$ and its connection to the dark matter. Remembering, the eleven dimensions in Witten's $M$-theory may be decomposed into the Lucas number $L_{5}=11=\varphi^{-5}-\varphi^{5}$. Reciprocity is indeed omnipresent in our world as piloting waves that accompany all observable earthen and cosmic matter. As a side effect of the $I R$ theory some fundamental constants such as the gyromagnetic factor of the electron, Sommerfeld s fine-structure constant as well as the charge of the electron must be marginally changed caused by altered relativistic corrections. Consequences also arise for our vision about the evolution of life and consciousness.
\end{abstract}

\section{Keywords}

Reciprocity, Reciprocal Lattice, Fourier Transform, Archimedes' Constant, 
Matter-Wave Duality, Pilot Wave, Golden Mean, E-Infinity Theory, Information Relativity Theory, Gyromagnetic Factor, Fine-Structure Constant, Quantum Entanglement, Dark Matter and Cosmos, Superconductivity

\section{Introduction}

Recently, the author reported on a reciprocity relation between the mass constituents of the universe and Hardy's maximum quantum entanglement probability of two quantum particles [1] [2] [3] [4]. Hardy's maximum probability was before connected with the dark energy question, using a fractal Cantorian set theory developed by El Naschie (E-infinity theory) [5] and further elaborated by Marec-Crnjac [6]. However, in the last years a step was made in direction of a unification of physical theories with the Information Relativity theory of Suleiman [7] [8]. Whereas great philosophers understood reciprocity as relation between action and reaction (Hegel [9]) or as connection between freedom and ethical necessities (Kant [10]), in this contribution a more mathematical as well as physical view on reciprocity was given. Because the term reciprocity has something in common with the concepts of duality and complementarity, their different meaning was shortly addressed in Appendix I.

The following chapter deals with the Fourier transform and the reciprocal lattice of crystallography that describes results of diffraction on crystals as a special kind of particle-wave duality. The reciprocity relation between boundary and enclosed area of a circle is shortly treated, followed by the reciprocity property of the golden ratio in a separate chapter. Then the fractal Cantorian set theory of $E I$ Naschie [5] and Marek-Crnjac was appreciated [6]. The final chapter illuminates consequences of the famous scale-free Information Relativity theory of Suleiman with its proven particle-wave duality, which explains for instance the double-slit experiment [11] and suggests dark energy as piloting waves of moving matter, touching up the old De-Broglie-Bohm theory [12] [13] [14]. The work was supplemented by thoughts of the author about further consequences of the $I R$ theory. It seems that the secrets of the electron, spin, tremor and the anomalous gyromagnetic factor $\left(g_{\mathrm{e}}\right)$ as well as electron pairing in superconductors [15] [16], can simply be explained or corrected with this new theoretical approach to demystifying the physics even more. The inferred Sommerfeld fine-structure constant $\alpha$ may be altered as well as the experimental $g_{\mathrm{e}}$ value, concerning the relativistic shift correction. Related to it, the charge of the electron has to be changed, too. In addition, a corrected version of the Niehaus EZBW (extended Zitterbewegung may serve as a probabilistic model for the electron [17]. Consequences also arise for our vision about the evolution of life and consciousness. The conjecture is that all things are interwoven with the reciprocal, where connection with the golden ratio indicates some system stability.

The particle-wave duality is also essential in the quantum information theory, 
where the unit of information is given by the quantum bit (qubit) coined by Schumacher [18], which exhibits the aspects of particle localization (counting) and wave interference to represent a signal with high fidelity [19]. Such two-state quantum system can be represented by the superposition principle:

$$
S U(2) \cong H_{1} \ni Q=z_{1}|Y e s\rangle+z_{2}|N o\rangle
$$

where $z_{1}$ and $z_{2}$ are complex numbers, and $z_{1}^{2}+z_{2}^{2}=1$ [19]. However, the quantum information aspect cannot be a main topic of this limited contribution.

\section{Fourier Transform and Reciprocal Space}

The diffraction of $X$-rays of sufficient wavelength on crystals leads to a characteristic diffraction pattern, where the electron density $\rho(\boldsymbol{r})$ of a crystal structure is transformed to a reciprocal lattice, weighted with intensities $I(\boldsymbol{h}) \propto|F(\boldsymbol{h})|^{2}$, where $F(\boldsymbol{h})$ represents structure amplitudes (Fourier coefficients) according to the transform

$$
F(\boldsymbol{h})=\int_{V} \rho(\boldsymbol{r}) \mathrm{e}^{2 \pi i h r} \mathrm{~d} V .
$$

The vectors $\boldsymbol{r}=x \boldsymbol{a}+y \boldsymbol{b}+z \boldsymbol{c}$ and $\boldsymbol{h}=h \boldsymbol{a}^{*}+k \boldsymbol{b}^{*}+\boldsymbol{l c}^{*}$ are position vectors of the crystal lattice respectively the reciprocal lattice of a diffraction pattern. The lattice parameters $\boldsymbol{a}, \boldsymbol{b}, \boldsymbol{c}$ respectively $\boldsymbol{a}^{*}, \boldsymbol{b}^{*}, \boldsymbol{c}^{*}$ are the repeat units along the lattice axes. The reader may follow a reciprocal lattice exercise in more detail, given as a lecture in [20].

The inverse Fourier transform, using the structure amplitudes as coefficients, delivers the electron density $\rho(\boldsymbol{r})$ of the crystal

$$
\rho(\boldsymbol{r})=\int_{V^{*}} F(\boldsymbol{h}) \mathrm{e}^{-2 \pi i h r} \mathrm{~d} V^{*},
$$

where $V^{*}$ is the reciprocal volume.

In this way, a crystal structure can be completely solved by means of a diffraction experiment.

However, because only intensities $I(\boldsymbol{h})$ are measured, phases of the structure amplitudes are lost and must be recovered by elaborated crystallographic methods.

Applying a transform such as the Fourier transform one goes from the object space to the image space or reciprocal space. If the original variable would be the time, then the transformed reciprocal variable would be a frequency, exemplified by the Laplace transform of electrical decay processes. Going from the object space to the reciprocal space one may heretically ask, what could be the Fourier transform of the entire cosmos, delivering an inverse universe or whatever else?

\section{Relationship between Boundary and Enclosed Area of a Circle}

Quoting the references [21] [22], the area enclosed by a circle of radius 1 yields

$$
A=\pi=4 \int_{0}^{1} \sqrt{1-x^{2}} \mathrm{~d} x,
$$


where $\pi$ is Archimedes constant, the well-known circle constant. One obtains the circumference $C$ by using the reciprocal of the integrand

$$
C=2 \pi=4 \int_{0}^{1} \frac{1}{\sqrt{1-x^{2}}} \mathrm{~d} x .
$$

This connection between the boundary and the enclosed area is of fundamental importance. It may be thought of as a geometrical analog to the more general matter-wave duality that is being treated below. Besides, Archimedes constant $\pi$ and the golden ratio $\varphi$ as the fractal numerical dominators of our existence show an intimate numerical connection, and we may ask, in which manner nature makes use of this [3] [23]. An elegant continued fraction representation of $\pi$ was given by Lange [24].

\section{Mushkolaj's Reciprocal Transition Temperatures}

In 2014 Mushkolaj [25] has presented a theory of critical temperatures for phase transitions such as superconductivity, using an elastic atomic collisions model as well as an elastic spring model. He found two inverse $T_{c}$ functions of the form

$$
T_{c}(\text { collision }) \propto\left(\sqrt{M_{1} \cdot M_{2}} \cdot \Delta x^{2}\right)^{-1}, T_{c}(\text { spring }) \propto \sqrt{M_{1} \cdot M_{2}} \cdot \Delta x^{2} \quad(5 \mathrm{a}, \mathrm{b})
$$

where $M_{1}, M_{2}$ are the colliding or by a spring connected masses, and $\Delta x$ is the distance between atoms respectively the spring stretch length in Hooke's region.

If we associate the atomic collision model with particles and the spring model with waves, we are faced with a reciprocity relation between the two excitation variants and again with the duality between particles and waves in a special form.

\section{The Golden Mean Beauty and Its Intrinsic Reciprocity Property}

The golden mean or golden ratio $\varphi$ is an omnipresent number in nature, found in the architecture of living creatures as well as human buildings, music, finance, medicine, philosophy, and of course in physics and mathematics [26] [27]. It is the most irrational number known and a number-theoretical chameleon with a self-similarity property. On the other hand, its infinite continued fraction representation is the simplest of all and is represented by [28]

$$
\varphi=\frac{\sqrt{5}-1}{2}=\frac{1}{1+\frac{1}{1+\frac{1}{1+\cdots}}}
$$

It impressively underlines the fractal character of this number. Most obviously, the golden mean mediates stability of a system, because only "particles" as the center of gravity of vibrations with most irrational winding survive. Important relations involving $\varphi$ are summarized below. However, to prevent confusion, in textbooks of mathematics the reciprocal value for $\varphi$ is frequently used. 


$$
\begin{gathered}
\varphi=\frac{\sqrt{5}-1}{2}=0.618033988 \cdots, \varphi^{-1}=1+\varphi=\frac{\sqrt{5}+1}{2}=1.618033988 \cdots \quad(7 \mathrm{a}, 7 \mathrm{~b}) \\
\varphi^{2}=1-\varphi=0.381966011 \cdots, \varphi^{-2}=2+\varphi=2.618033988 \cdots \quad(8 \mathrm{a}, 8 \mathrm{~b}) \\
\left(\varphi^{2}+1\right)^{-1}=\left(\varphi^{-2}+1\right) / 5 \\
\text { or equivalently }\left(\varphi^{-2}+1\right)^{-1}=\left(\varphi^{2}+1\right) / 5 \\
\varphi^{5}=\frac{1-\varphi}{1+\varphi} \varphi^{2}=0.090169943 \cdots \\
\frac{\varphi^{5}}{2}+\frac{5}{2} \varphi^{2}=1 \\
\varphi^{3}+2 \varphi^{2}=1
\end{gathered}
$$

Hardy's maximum quantum entanglement probability of two quantum particles [1] [2] exactly equals the fifth power of $\varphi$ (Figure 1). This asymmetric probability distribution function with $p_{\tau}$ as entanglement variable, running from not entangled states to completely entangled ones, is given by

$$
P=p_{\tau}^{2} \frac{1-p_{\tau}}{1+p_{\tau}}
$$

This function, displayed in Figure 1, turns out to be a central topic of the Information Relativity theory of Suleiman [7] [8] by mapping the transformation of his relative energy density (see Chapter 9 and compare the red curves in Figure 3 and Figure 4).

The probability function according to Equation (13) can be recast to an adapted distribution by means of a varied Fisher transformation (Figure 2) [29]

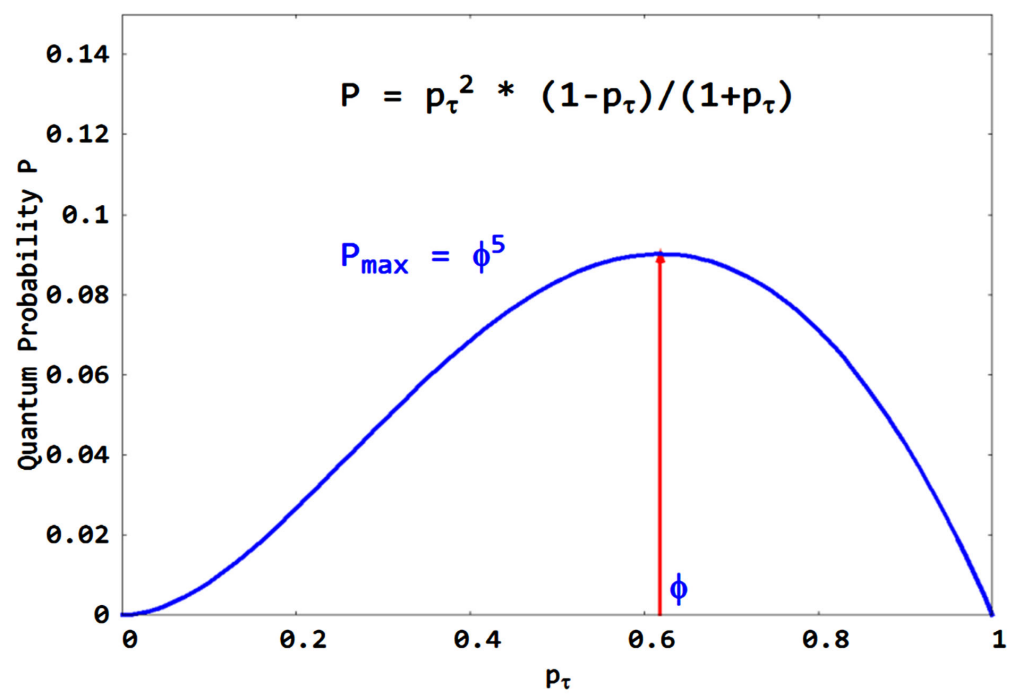

Figure 1. Hardy's quantum probability $P$ for two particles [1], where $p_{\tau}$ can be thought of as entanglement variable, running from not entangled states to completely entangled ones. Compare it with the equivalent red curve in Figure 3 [3] [7]. 


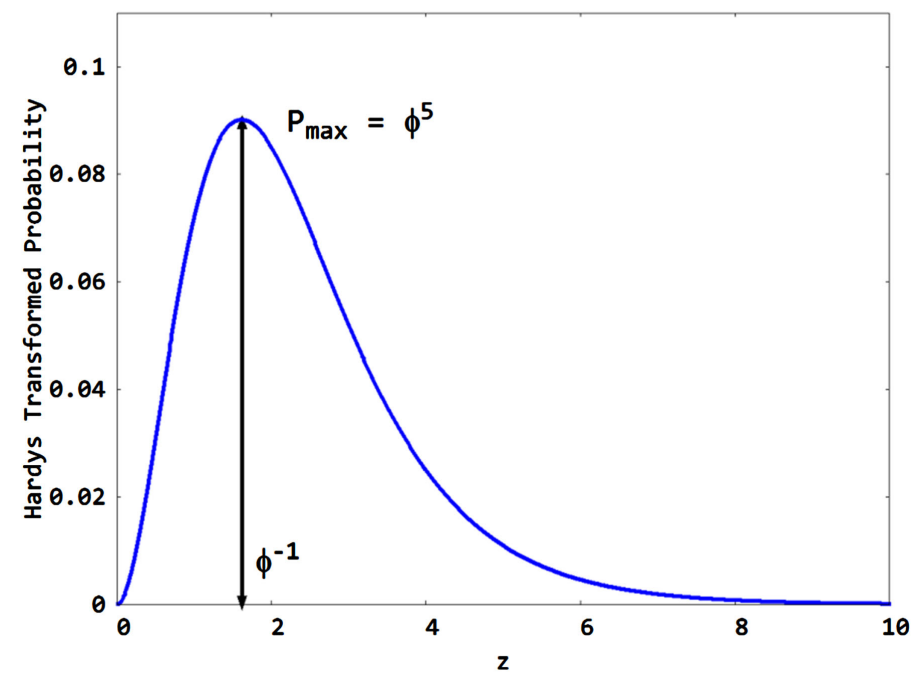

Figure 2. Fisher transform of Hardy's probability function, representing an asymmetrical distribution with its maximum now at $z=\varphi^{-1}$ (see Equation (15) and Figure 3).

$$
z=\frac{\sqrt{5}}{2} \ln \left(\frac{1+p_{\tau}}{1-p_{\tau}}\right)=\sqrt{5} \cdot \operatorname{artanh}\left(p_{\tau}\right)
$$

where the pre-factor was chosen as $a=\frac{\sqrt{5}}{2}=\varphi+\frac{1}{2}$. Then one gets for $f(z)$

$$
f(z)=\left(\frac{\exp \left(\frac{z}{a}\right)-1}{\exp \left(\frac{z}{a}\right)+1}\right)^{2} \exp \left(\frac{z}{a}\right)^{-1}
$$

A comparison is made between the curves displayed in Figure 1 and Figure 2 with the result of the $I R$ theory in Figure 3, but in logarithmic representation.

Moreover, in a subsequent contribution a geometric analog to Hardy's probability function will be presented with significance, besides geometry, to crystallography, electrostatic, botany, coding theory and other disciplines.

Infinite continued fraction representations of $\varphi^{5}$ and its inverse yield

$$
\begin{gathered}
\varphi^{5}=\frac{1}{11+\frac{1}{11+\frac{1}{11+\cdots}}} \\
\varphi^{-5}=11+\varphi^{5}=11+\frac{1}{11+\frac{1}{11+\frac{1}{11+\cdots}}}
\end{gathered}
$$

We notice that $L_{5}=11$ is a Lucas number. It results from the definition

$$
L_{n}=\varphi^{-n}+(-\varphi)^{n}
$$

The $L_{n}$ number series was named after the French mathematician François Édouard Anatole Lucas (1842-1891). 


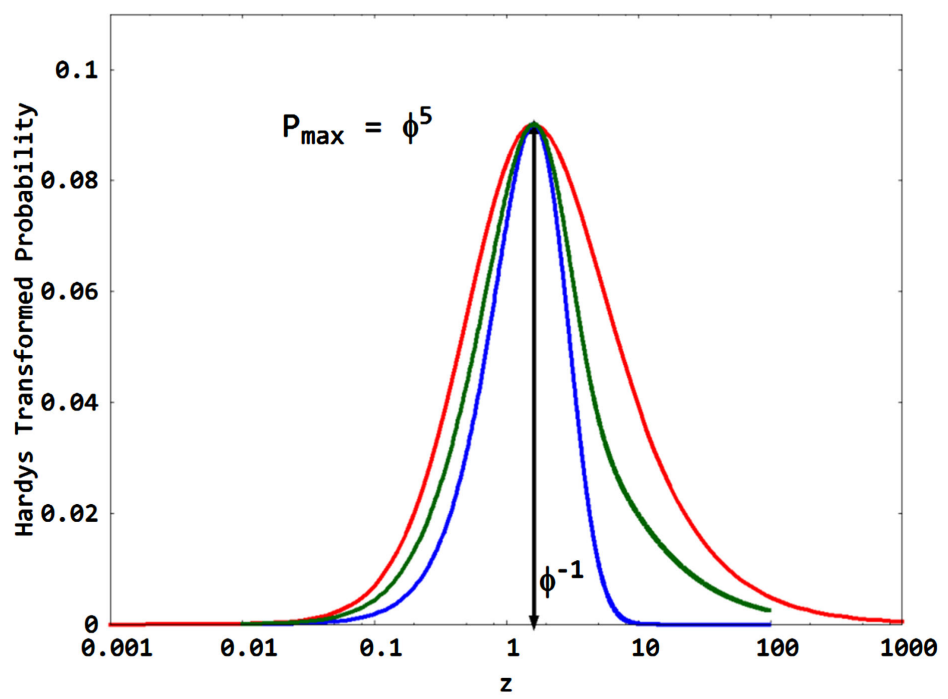

Figure 3. Fisher transform [29] of Hardy's probability function (blue curve) [1], compared with the more convincing, but nevertheless slightly asymmetric redshift representation of the energy density according to the information relativity theory (red curve) [8] (see also Figure 4). The green curve is a further adaption in direction of the red curve.

Many researchers have found the golden ratio to be important by trying to uncover secrets of the universe and its mass respectively energy distribution [3]-[8]. In the next Chapters this significance will be demonstrated.

If one deals with exponential functions, the author has learnt from Sherbon [25] that the Lambert $W$-function can serve as an analog to the golden ratio for exponential functions. Again, it is shown a sort of reciprocity, if one writes the relation as follows

$$
\exp (W(z))=W(z) / z
$$

Especially is

$$
\begin{gathered}
\Omega=W(1)=\exp (-W(1))=0.5671432904 \cdots \\
W(2)=\frac{1}{2} \exp (-W(2))
\end{gathered}
$$

and

$$
W\left(\frac{1}{2}\right)=2 \cdot \exp \left(-W\left(-\frac{1}{2}\right)\right)
$$

The quoted publication of Sherbon [25] is highly interesting for all researchers, who want to learn more about of the fundamental nature of Sommerfelds fine-structure constant.

\section{Golden Ratio, Archimedes' Constant and Sommerfeld's Fine-Structure Constant}

Often you wonder why our world is what it is. Fundamental numbers such as the golden ration $\varphi$, the circle constant $\pi$ as well as Sommerfeld s fine-structure constant $\alpha$ and their obvious similarities play an important role. Some approxima- 
tions should illustrate it. So one can connect the number $\pi$ with the reciprocal of the Sommerfeld constant $\alpha^{-1} \approx 137$ [23] [27] or with Hardy's quantum probability $\varphi^{5}$ [23].

$$
\begin{gathered}
\pi \approx 3+\frac{16}{137-24}=3.1415929 \cdots \\
\frac{\pi-3}{\pi}=0.04507 \cdots \approx \frac{\varphi^{5}}{2}=0.04508 \cdots \\
\frac{3}{\pi}=0.9549296 \cdots \approx \frac{5}{2} \varphi^{2}=0.954915 \cdots \\
\alpha^{-1}=137+\frac{2}{5} \varphi^{5}=137.0360 \cdots
\end{gathered}
$$

These approximations, believed to be accidental by others, may find now and then application in the following discussions.

\section{Golden Mean and Madelung Constant for a Rocksalt-Type $2 D$-Lattice}

In a previous publication the author drew attention to the numerical similarity between the golden mean and the Madelung constant [30] for a two-dimensional rocksalt-type lattice [4].

The Madelung constant $\alpha_{2 D}$ was iteratively determined with very high precision by Triebl [31] giving

$$
\alpha_{2 D-\mathrm{NaCl}}=1.615542626711299 \ldots
$$

The $\alpha_{2 D}$ value is very close to the quotient of two Fibonacci numbers, 21/13= $1.615385, \ldots$ and can be adapted to $\varphi^{-1}$ by only slight distortion of the square net along the two dimensions or by involving the third one to allow a quite flat curvature [4].

The difference to the inverse of the golden mean $\varphi^{-1}$ is only marginal and gives

$$
\varphi^{-1}-\alpha_{2 D-\mathrm{NaCl}}=0.002491362 \cdots
$$

This almost numerical equality was applied to Villata's lattice universe [32] consisting of matter and antimatter with gravitational charges of opposite sign at positions of a $2 D$ rocksalt-type lattice. Then the ratio of repulsive contribution to the attractive one gives

$$
1+\alpha_{2 D} \approx 1+\varphi^{-1}=\varphi^{-2}
$$

This relation leads the author to a proposal for the golden mean based calculation of the mass constituents of the universe [4] independent from other approaches and with only marginal differences to such results [5] [6].

A certain reciprocity property may be suggested for a two dimensional rocksalt-type matter-antimatter lattice independent of whether it is a real possibility. The question is whether a conditionally flat lattice universe with a Madelung constant of $\varphi^{-1}$ would guarantee sufficient stability to exist over long periods of time. 


\section{Golden Mean as Dimension of Empty Set and Zero Set}

El Naschie's E-infinity ( $\left.\varepsilon^{\infty}\right)$ theory [33], not commonly known or accepted by physicists, originates from a fractal Cantorian set theory [34] as a number-theoretical route of physics for explaining the dualism between particles and waves that can help solving cosmological mysteries such as dark matter and dark energy [35]. The quantum particle $P_{Q}$ is symbolized by the bi-dimension of the zero set, while the guiding wave $W_{Q}$ surrounding the quantum particle is given by the bi-dimension of the empty set according to

$$
\operatorname{dim}(X)=\left(n, d_{c}^{(n)}\right)
$$

where $n$ is the Urysohn-Menger topological dimension [36] [37] and

$$
d_{c}^{(n)}=\left(\varphi^{-1}\right)^{n-1}
$$

represents the Hausdorff dimension [38], where $\varphi$ is the golden mean as defined before.

$$
\begin{aligned}
& \text { It results for } P_{Q} \operatorname{dim}\left(P_{Q}\right)=(0, \varphi) \\
& \text { respectively for } W_{Q} \operatorname{dim}\left(W_{Q}\right)=\left(-1, \varphi^{2}\right)
\end{aligned}
$$

By using these dimensions a probabilistic quantum entanglement calculation [6] [33] with velocity restriction $v \rightarrow c$ delivers effective quantum gravity formulas for the cosmological mass (energy) constituents of baryonic matter $e_{M}$, dark matter $e_{D M}$, entire dark constituents $e_{E D}$, and pure dark energy $e_{P D}$ as follows

$$
\begin{gathered}
e_{M}=\frac{1}{2} \frac{1-\varphi}{1+\varphi} \varphi^{2}=\frac{\varphi^{5}}{2}=0.04508497 \\
e_{E D}=1-e_{M}=\frac{5}{2} \varphi^{2}=0.9549150 \\
e_{D M}=\frac{3}{2} \varphi^{4}=0.218847 \\
e_{P D}=2 \varphi-\frac{1}{2}=0.736068 \\
e_{M}+e_{D M}+e_{P D}=1
\end{gathered}
$$

Recasting the matter amounts into a suitable form,

$$
e_{M}=\frac{1}{10} 5 \varphi^{5}, e_{D M}=\frac{1}{10}\left(5 \varphi^{5}\right)^{-1}=0.2218
$$

a reciprocity relation was confirmed between $e_{M}$ and $e_{D M}$ giving a persuasive equation for the pure dark energy [3]

$$
e_{P D}=1-\frac{1}{10}\left(5 \varphi^{5}+\left(5 \varphi^{5}\right)^{-1}\right)=0.7331(73.31 \%)
$$

Such quantum entanglement based coincidence means that the constituents of the cosmos should not be considered independent of each other, which was confirmed by the $I R$ theory. 
Importantly, if one compares the results given here with the following ones of the information relativity $(I R)$ theory, then El Naschie's set theoretical approach is restricted to $v \rightarrow c$, whereas the more general $I R$ theory delivers results for the recession velocity $\beta=\frac{v}{c}$ in the hole range $0 \leq \beta \leq 1$ ( $c$ is the speed of light).

\section{Information Relativity Theory of Suleiman and Golden Mean}

Many formal explanations or physical constructs that bothered long time the world of physics are overcome by the new exciting Information Relativity theory, developed by Suleiman [7] [8]. It is not the intention of the author to keep the reader away from studying this theory in detail for himself. Therefore, only a sparse introduction was given. Suleiman found an overlooked flaw in Newton's physics and corrected physical processes for time displacements between observer and moving bodies. Transformations for time duration, length, mass density as well as energy density were applied to a whole bunch of physical phenomena, which could be explained now in simple and beautiful clarity. For instance, Suleiman derived for the matter energy density $e_{M}$ of a moving body with velocity $v$ and rest density $\rho_{o}$

$$
e_{M}=\frac{1}{2} \rho v^{2}=\frac{1}{2} \rho_{o} c^{2} \frac{1-\beta}{1+\beta} \beta^{2}=e_{o} \frac{1-\beta}{1+\beta} \beta^{2},
$$

where $\beta=\frac{v}{c}$ is the recession velocity respectively $e_{o}=\frac{1}{2} \rho_{o} c^{2}$.

The matter energy density reached its maximum at a recession velocity of $\beta=\varphi$. Replacement of this special value in Equation (41) gives

$$
\left(e_{M}\right)_{\max }=e_{o} \frac{1-\varphi}{1+\varphi} \varphi^{2}=e_{o} \varphi^{5}=e_{o} \cdot 0.09016994 \cdots
$$

Remembering, $\varphi^{5}$ represents Hardy's quantum probability at the maximum. This result was commented by the author in a publication before mentioned [3].

Suleiman aptly characterized the behavior at the critical point $\beta_{\mathrm{cr}}=\varphi$ as phase criticality at cosmic scale [8]. The dark matter density transforms as

$$
\frac{e_{D M}}{e_{o}}=\frac{2 \beta^{3}}{1+\beta}
$$

The relations are depicted in Figure 4. If one calculates the energy density amounts (ratios) of matter and dark matter contributions at this point, one gets again a golden mean representation like Russian dolls nesting

$$
\varphi^{3}+2 \varphi^{2}=0.236067976 \cdots+0.763932023 \cdots=1
$$

The difference gives $2 \varphi^{2}-\varphi^{3} \approx(\sqrt{2}-1) \frac{4}{\pi}$.

The case, where according to the Information Relativity theory of Suleiman [7] [8] just at the recession velocity of $\beta=1 / 3$ the matter and the dark matter density will be the same, delivers for the density amount the reciprocal of another Lucas number, namely $L_{6}=18$ (see Chapter 5 and Figure 4) 


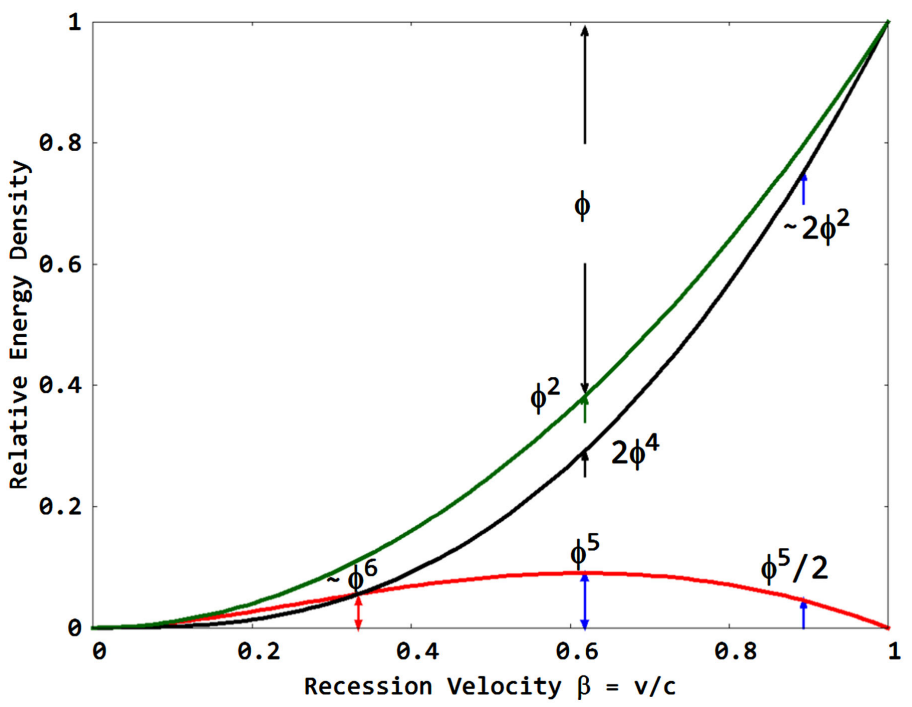

Figure 4. Golden mean dominance in the evolvement of the energy density with the recession velocity according to the information relativity theory of Suleiman [7] [8]. $\Phi^{5}$ represents Hardy's maximum quantum probability. Red curve: matter energy density, black curve: dark matter energy density, green curve: energy density sum.

$$
e_{M}=e_{D M}=L_{6}^{-1}=\frac{1}{18}=0.055555 \cdots=\left(\varphi^{6}+\varphi^{-6}\right)^{-1} \approx 0.055728089 \cdots=\varphi^{6}
$$

Furthermore, if the recession velocity at $\beta_{e q}=1 / 3$ is mirrored at $\beta_{\mathrm{cr}}=\varphi$, it resulted $\beta_{\text {mir }}=0.9027$. In its vicinity at $\beta=0.89297$ the matter energy density would be exactly $\varphi^{5} / 2=0.04508497 \ldots$ respectively the dual dark component $0.7523 \cdots \approx 0.763932 \cdots=2 \varphi^{2}$ (Figure 1$)$.

It approximately indicated a situation that is elaborated for $v \rightarrow c$ by means of the fractal set theory summarized before in Chapter 8 .

In Figure 5 the energy densities were illustrated via the redshift, which reads as $z=\beta /(1-\beta)$. It is suggested to fit the only slightly asymmetric red curve with the aid of a Cauchy function of exotic non-integer order on the basis of the golden mean introduced by the present author some time ago [39].

Suleiman's $I R$ theory validates once more the importance of the golden mean in solving physical phenomena. Reciprocity is given by the proposed duality between particle and wave.

As was demonstrated by Suleiman (Figure 6), an increase of the redshift $Z$ caused the matter density of the travelling corpuscular particle successively to diminish, while energy is transformed into the wave-like dark component and vice versa [8]. This supports elegantly the concerns of the work here presented.

\section{Mystery of the Electron and Golden Mean}

The electron, considered as center of compacted information, still keeps its secrets, but not for long. Whereas the hydrogen atom problem was just solved by Suleiman [8] without any assumption of quantization of the electron's orbits and using $I R$ transformation of length as 


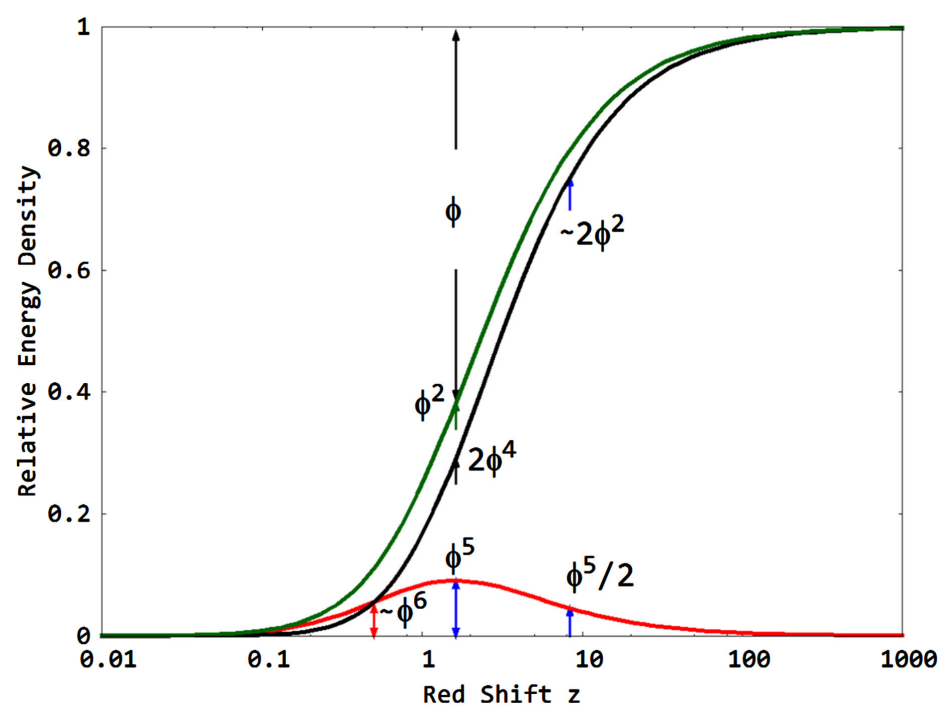

Figure 5. Energy densities related to the red shift $z=\beta /(1-\beta)$ (logarithmic scale) according to Suleiman [8]. Colored curves have the same meaning as in Figure 1. Now the coincidence point is at $Z=1 / 2$.

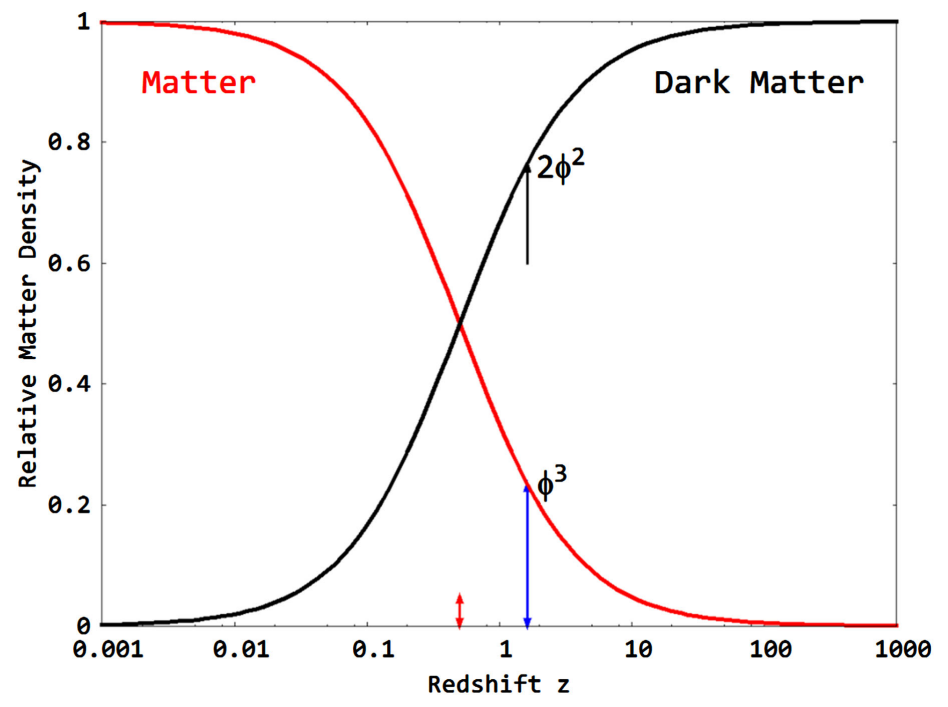

Figure 6. Suleiman's famous reciprocal (complementary) duality between matter density and dark matter one. Logarithmic scale, red arrow at $z=1 / 2$, blue arrow at $z=\varphi^{-1}=$ $1.61803398 \cdots$ (see also Equation (39)). Applied matter density transformations in terms of the redshift [8]: $\frac{\rho_{M}}{\rho_{0}}=\frac{1}{2 z+1}, \frac{\rho_{D M}}{\rho_{0}}=\frac{2 z}{2 z+1}$.

$$
\frac{l}{l_{0}}=\frac{1+\beta}{1-\beta}
$$

other constructs like the electron spin [25] or the measured anomalous gyromagnetic factor of the electron may be solved fractal-deterministic, supported by application of $I R$ transformations. Also the fractal nature of electron pairing in superconductors should be reassessed this way.

The $g_{e}$ factor of the electron, conceived as a classical charged particle, is de- 
termined by the relation

$$
\vec{\mu}=g_{e} \mu_{B} \frac{\vec{S}}{\bar{h}}, \mu_{B}=\frac{e}{2 m}
$$

where $\vec{\mu}$ is the observable magnetic moment, $\mu_{B}$ is the Bohr magneton, and $\vec{S}$ is the spin of the electron, $e$ respectively $m$ are charge respectively mass of the electron, and $\bar{h}$ is the reduced Planck constant.

However, the spin as half-integer quantum number of the electron was introduced without any physical justification [40]. Very recently, a first attempt has been undertaken by $\mathrm{He}$ et al. [41] to connect the golden mean with the ad hoc spin-1/2 construct. Such golden mean approach may be the result of dark halo movement around the stretched electron in the sense of the Information Relativity theory.

Remembering that the "anomalous part" of the gyromagnetic factor $\Delta g_{e}$ was recently given by a simple and solely golden mean representation with sufficient accuracy [42]

$$
\Delta g_{e}=\ln \left(1+\frac{\varphi^{6}}{24}\right)=0.002319312 \cdots
$$

while a series expansion yields a value more accurate up to the tenth decimal place

$$
g_{e}=2+\frac{\varphi^{6}}{24}-\frac{1}{2}\left(\frac{\varphi^{6}}{24}\right)^{2}-\frac{1}{4}\left(\frac{\varphi^{6}}{24}\right)^{3}=2.002319304 \cdots
$$

This result may be compared to the high accuracy of the best known experimental value for $g_{e}$ determined as one-electron cyclotron transition for an electron trapped in an electrostatic quadrupol potential (Penning trap) [43]

$$
g_{e}=2.00231930436182(52)
$$

In a subsequently presented seminal idea of $\mathrm{He}$ et al. [41] the spin quantum number $s$ in the spin momentum term $\frac{\vec{S}}{\bar{h}}=\sqrt{s(1+s)}$ was replaced by a quantized golden mean $\tilde{\varphi}$ giving

$$
\frac{g_{e}}{2}=\sqrt{\tilde{\varphi}(1+\tilde{\varphi})}
$$

with the value $\tilde{\varphi}=0.6190713336307(34)$ as He-Chengtian average [44] [45] to adapt the full accuracy of $g_{e} / 2$. One can calculate $\tilde{\varphi}$ by a very simple formula, which resembles the representation for $\varphi$ (Equation $7(\mathrm{a})$ ) and delivers exactly the given value

$$
\tilde{\varphi}=\frac{1}{2}\left(\sqrt{5+\left(g_{e}+2\right)\left(g_{e}-2\right)}-1\right)=\frac{1}{2}\left(\sqrt{1+g_{e}^{2}}-1\right)
$$

and for the $I R$ corrected value of $g_{e}=2.0023190900$ (see Chapter 11)

$$
\tilde{\varphi}=0.619071237 \cdots
$$

Using this formula, the gyromagnetic factor resulted simply as function of $\alpha / \pi$ 
[46]

$$
\begin{gathered}
g_{e} \approx 2 \sqrt{1+\frac{v_{K}}{2 \pi c}}=2 \sqrt{1+\frac{\alpha}{\pi}}=2.00232147 \\
\text { giving } \tilde{\varphi}=0.619072302 \ldots
\end{gathered}
$$

where $V_{K}$ is the Klizing speed and $c$ the speed of light.

The latest released values of Sommerfeld 's fine-structure constant $\alpha$ [47] respectively its reciprocal value is quoted according to NIST [48] being

$$
\begin{aligned}
& \alpha=0.0072973525693(11) \\
& \alpha^{-1}=137.035999084(21)
\end{aligned}
$$

An approximation using the $\alpha / \pi$ series expansion yielded

$$
\begin{aligned}
& g_{e} \approx 2 \sqrt{1+\frac{\alpha}{\pi}-\frac{1}{2}\left(\frac{\alpha}{\pi}\right)^{2}}=2.002318778 \cdots \\
& \text { or } g_{e} \approx 2 \sqrt{1+\ln \left(1+\frac{\alpha}{\pi}\right)}=2.002318782 \ldots \\
& \tilde{\varphi}=\frac{1}{2}\left(\sqrt{5+4 \cdot \ln \left(1+\frac{\alpha}{\pi}\right)}-1\right) \\
& \text { and further } \\
& =\frac{1}{2}\left(\sqrt{5+\ln \left(1+\frac{\alpha}{\pi}\right)^{4}}-1\right)=0.619071099 \cdots
\end{aligned}
$$

The deviation between this a bit underdetermined $\tilde{\varphi}$ value and the newly relativistic corrected one is in the seventh decimal place as well as the corresponding $g$-factors. It is hoped that precisely re-determined experimental factors may lessen these deviations further.

One may ask, what the infinitely continued fraction representation of $\tilde{\varphi}$ would result in. We can write similar to the golden mean [28]

$$
\tilde{\varphi}=\frac{\sqrt{5+\delta}-1}{2}=\frac{1}{1-\delta_{1}+\frac{1}{1-\delta_{1}+\frac{1}{1-\delta_{1}+\cdots}}}
$$

The calculation with $\delta_{1}=0.00374774 \approx \frac{1}{266 . \overline{6}} \approx \frac{\delta}{\varphi} \approx \frac{\varphi^{5}}{4 !}$ yielded $\tilde{\varphi}=0.619071096$. Indeed, the number $266 . \overline{6}$ is very interesting. Division of this number by integers frequently delivers numbers with repeating decimals, exemplified by $266 . \overline{6} / 24=11 . \overline{1}$. If one associates this number with rounds, then one would need 27 ones to complete 20 -times the full 360 degrees extent.

With an assumed involvement of the fifth power of the golden mean in the continued fraction representation one may speak of a nested golden mean representation. This result supports once more the fractal-deterministic approach chosen for the physics of the electron beyond the ad hoc half-spin assumption, characterizing the electron as complexly nested resonating entity. An alternating approach for the gyromagnetic factor is given in Appendix II. 


\section{Alteration of Fundamental Constants}

The calculation of the electron's gyromagnetic factor is the prime example for application of the $Q E D$. However, a cascade of Feynman diagram calculation must be done to determine the pre-factors of systematic perturbative expansions in powers of $\alpha / \pi$ [46] [49]. It is not so long ago that Gabrielse et al. asked "whether it is likely that other adjustments of the $Q E D$ theory will shift the $\alpha$ that is determined from the electron $g$ ?" and answered "we hope not" [50]. Nevertheless, the $Q E D$ theory should be corrected for $I R$ transformations to iron out some flaws, and the author suggests a considerable simplification of $Q E D$ calculations as a renewed successful tool, altering the inferred $\alpha$ constant, and related to it, the charge of the electron. Also importantly, the experimental value of $g$ must be corrected, too. The applied relativistic shift of the cyclotron frequency $\omega_{c}=\frac{e B}{\gamma m} \quad\left(\omega_{c}=\right.$ cyclotron frequency, $B=$ magnetic field strength in Tesla $)$ was performed using the familiar relativistic factor $\gamma$. However, $\gamma$ should be replaced by the mass transformation according to the $I R$ theory [8]

$$
\frac{m}{m_{0}}=\frac{1-\beta}{1+\beta}
$$

For the classical case the corrected frequency $\omega_{c}$ is

$$
\omega_{c}=\omega_{0}\left(1-\frac{E_{n}}{m c^{2}}\right)
$$

where the energy $E_{n}$ of the $n$th quantum state of a harmonic cyclotron oscillator is given as

$$
E_{n}=\left(n+\frac{1}{2}\right) \bar{h} \omega_{c}
$$

The classical relativistic shift $\delta$ in the cyclotron frequency per energy quantum was approximated by the level spacing of the harmonic oscillator giving [51]

$$
\delta=-\bar{h} \omega_{c}^{2} /\left(m c^{2}\right)
$$

For the $I R$ theory one yields a much greater and positive shift because the cyclotron frequency yields now

$$
\omega_{c} \approx \omega_{0} \cdot\left(1+2 \cdot \sqrt{\frac{2 E_{n}}{m c^{2}}}\right)
$$

The relativistic shift $\delta$ is approximated by

$$
\delta \approx \frac{\mathrm{d} \omega_{c}}{\mathrm{~d} n}=2 \omega_{0} \sqrt{\frac{2}{m c^{2}}} \cdot \frac{\mathrm{d} \sqrt{E_{n}}}{\mathrm{~d} n}=\omega_{0} \sqrt{\frac{2}{m c^{2} E_{n}}} \cdot \bar{h} \omega_{c}=2 \cdot \omega_{0} \sqrt{\frac{2 E_{n}}{m c^{2}}}
$$

The gyromagnetic factor as $g / 2$ can be determined from the observed eigenfrequencies [51]

$$
\frac{g_{e}}{2}=1+\frac{\bar{\omega}_{a}-\bar{\omega}_{m}}{\bar{\omega}_{c}-\bar{\omega}_{m}}
$$

where the $\bar{\omega}$ values are marginally modified with respect to the free-space values, $\omega_{a}$ is the anomalous frequency, and the spin frequency is $\omega_{s}=\omega_{c}+\omega_{a}$, 
$\frac{\bar{\omega}_{z}^{2}}{2 \bar{\omega}_{c}}=\bar{\omega}_{m}$ is the magnetron frequency, using the dip frequency $\omega_{\mathrm{z}}$ in $H z$ [51].

For the experimentally chosen cyclotron frequency of $v_{\mathrm{c}}=149.2 \mathrm{GHz}$, the classical relativistic shift is calculated to be $\delta=-2 \pi \cdot 182.1 \mathrm{~Hz}$ compared to the $I R$ corrected one giving $\delta=+2 \pi \cdot 14.78 \mathrm{Mhz}$. One can estimate that $g_{e}$ becomes noticeably smaller by a factor of approximately 1.0001, meaning a correction of $g_{e}$ from the seventh decimal point downwards to about $g_{e} \approx 2.00231909$ ?

Now the scientific community is waiting for a most precise redetermination of the $g$-factor as well as the related Sommerfeld constant by experts [49] [51]. The aforementioned Zitterbewegung approach of Niehaus [17] should be revised by that author himself. The comment of the present author may have fulfilled its true purpose, if research on this topic proceeds well with application of the $I R$ theory [52].

\section{Fractal Superconductivity}

Nature presents much more relationships to keep in mind, where the golden mean is involved, and superconductivity is no exception. However, we must reassess the theory considering the dark matter surrounding the moving electrons, which dive into the dark after marriage, or in other words, become superconducting under special conditions. Before a golden ratio in the spin dynamics of the quasi-one-dimensional Ising ferromagnet $\mathrm{CoNb}_{2} \mathrm{O}_{8}$ was experimentally verified next a phase critical point by Coldea et al. [53], the present author suggested linking the optimum hole doping $\sigma_{0}$ of high- $T_{\mathrm{c}}$ superconductors with the golden mean in the form of Hardy's maximum quantum probability of two particles [15]

$$
\sigma_{0} \approx \frac{8}{\pi} \varphi^{5}=0.2293
$$

Obviously, this optimum is again near a quantum critical point in the phase diagram. In addition, the relation of the Fermi speed to the Klitzing speed comes out as

$$
\frac{v_{F}}{v_{K}} \approx \frac{2}{\pi} \varphi^{5}=0.0571
$$

Both relations document the fractal nature of the electronic response in superconductors. It was suggested recently that the same is true for conventional superconductors [16]. Also Prester had reported before about evidence of a fractal dissipative regime in high- $T_{\mathrm{c}}$ superconductors [54].

Interestingly, some time ago the present author connected the optimum transition temperature $T_{\text {co }}$ of high- $T_{\mathrm{c}}$ superconductors with a Fibonacci number $f_{\mathrm{i}}$, proportional to a domain width, by the relation $T_{\text {co }}=12,000 / f_{\mathrm{i}}[15]$. One yields the integer number 45, again as a product of solely Fibonacci numbers, when dividing this number by the number $266 . \overline{6}$ (see Chapter 10).

Quantum entanglement of two moving electrons may be influenced by local interaction of their interwoven dark matter surroundings, quoting the cogwheel 
picture of Suleiman [8]. What happens, if two stretched electrons locally interact to become superconducting? May such particle stretching in the end lead to a double-helically wounded wavy entity, which escapes in the dark? Nature is known to copy itself again and again. So the double-helix approach is not only beautiful, if we quote Gauthier's proposed entangled double-helix superluminal photon model [55]. Therefore, a model calculation for superconducting electron strands is suggested based on this idea, addressing the problem of left and right (mirrored) strands as well as objections against an apparent superluminal velocity. The double helix strand in nature is a special fabric of duality.

\section{Evolution of Life}

Nature repeatedly applied its building plans, based on the hierarchical golden number system, from largest to smallest dimensions, from the cosmos to the smallest living cell. Inasmuch the golden ratio is involved, reciprocity is considered as a vital element of life. Recently, thoughts to the link between cosmology and biology are impressively formulated [56]. Self-similarity as an element of chaos is intimately connected with self-organization of life producing compacted information and consciousness. However, visions about life such as Englands provocative approach of dissipation-driven adaption [57] or Pitkänen's formulation [58] suffer from not considering duality of particle and wave or reciprocity of matter and dark matter [8] and should be adapted to the new physics.

The evolution of life may take place similar to the statistical bootstrap model of colliding heavy particles, so the Hagedorn temperature $T_{H}$ comes into play. I quote the formulation of Rafelski and Ericson [59] to explain this: "When a drop of particles and resonances is compressed to the 'natural volume', it becomes another more massive resonance. This process then repeats, creating heavier resonances, which in return consist of resonances, and so on".

This nesting looks like a Menger sponge [56] [60]. The process could explain the evolution of life with $T_{H}$ around ambient temperatures.

With respect to the entire energy density of $\varphi^{2}$ at the phase critical point $\beta=\varphi$ one may suggest formulating the Hagedorn temperature $T_{H}$ proportional to the squared golden mean $\varphi$, where $\alpha^{\prime}$ is formally the tension of a string.

$$
T_{\text {Hag }} \approx \varphi^{2} \cdot \frac{1}{\alpha^{\prime}}
$$

It remains to interpret the not liked string tension by a more appropriate thermodynamic quantity at ambient equilibrium conditions.

\section{Conclusion}

The duality between a compacted entity and its surrounding in general as well as the duality between a moving particle or body and the accompanying wave or reciprocity between matter and dark matter is the very spice of life. This was proven by the beautiful information relativity theory of Suleiman. Reciprocity is impressively formulated by the words of Wolfgang Pauli: "God made the bulk; 
surfaces were invented by the devil" (quoted from [56]). As a consequence of the $I R$ theory some natural constants such as the gyromagnetic factor of the electron, Sommerfeld 's fine-structure constant as well as the charge of the electron are proposed to be marginally altered. The interpretation of superconductivity is influenced by the $I R$ approach, too. Also the evolution of life may find a new basis. If we have fully understand the new $I R$ physics with its particle-wave reciprocal dualism and intrinsic harmony, then we can shape our environment more effectively to achieve a balance between plants, animals and human beings, which enables a long-term life for all of us on earth. In this sense the golden mean should provide more beauty than chaos.

\section{Acknowledgements}

The author appreciated the critical reading of the manuscript by Prof. Ramzi Suleiman, University of Haifa, and the Triangle Research and Development Center (TCRD), who enriched the scientific community with his famous information relativity theory. The author is also grateful for the constructive criticism of a very creative reviewer.

\section{Conflicts of Interest}

The author declares no conflicts of interest regarding the publication of this paper.

\section{References}

[1] Hardy, L. (1993) Physical Review Letters, 71, 1665-1668. https://doi.org/10.1103/PhysRevLett.71.1665

[2] Mermin, N.D. (1994) American Journal of Physics, 62, 880-887. https://doi.org/10.1119/1.17733

[3] Otto, H.H. (2018) World Journal of Condensed Matter Physics, 8, 30-35. https://doi.org/10.4236/wjcmp.2018.82003

[4] Otto, H.H. (2018) Journal of Modern Physics, 9, 1-13. https://doi.org/10.4236/jmp.2018.91001

[5] El Naschie, M.S. (2013) Journal of Quantum Information Science, 3, 57-77. https://doi.org/10.4236/jqis.2013.32011

[6] Marek-Crnjac, L. (2013) Cantorian Space-Time Theory. Lambert Academic Publishing, Saarbrücken, 1-50.

[7] Suleiman, R. (2018) World Journal of Condensed Matter Physics, 8, 130-155. https://doi.org/10.4236/wjcmp.2018.83009

[8] Suleiman, R. (2019) Relativizing Newton. Nova Scientific Publisher, New York, 1-207.

[9] Inwood, M.J. (1995) A Hegel Dictionary. Blackwell Reference, Oxford, U.K.

[10] Caygill, H.A. (1992) A Kant Dictionary. Blackwell Reference, Oxford, U.K.

[11] Suleiman, R. (2016) A Relativistic Model of Matter-Wave Duality Explains the Result of the Double-Slit Experiment. 5th International Conference on New Frontiers in Physics, Zurich, 6-14 July 2016, 1-14. 
[12] De Broglie, L. (1923) Nature, 112, 540. https://doi.org/10.1038/112540a0

[13] Bohm, D. (1952) Physical Review, 85, 166-179. https://doi.org/10.1103/PhysRev.85.166

[14] Bohm, D. (1952) Physical Review, 85, 180-193. https://doi.org/10.1103/PhysRev.85.180

[15] Otto, H.H. (2016) World Journal of Condensed Matter Physics, 6, 244-260. https://doi.org/10.4236/wjcmp.2016.63023

[16] Otto, H.H. (2019) World Journal of Condensed Matter Physics, 9, 22-36. https://doi.org/10.4236/wjcmp.2019.91002

[17] Niehaus, A. (2017) Journal of Modern Physics, 8, 511-521. https://doi.org/10.4236/jmp.2017.84033

[18] Schumacher, B. (1995) Physical Review A, 51, 2738-2747. https://doi.org/10.1103/PhysRevA.51.2738

[19] Ionescu, L. (2008) On the Arrow of Time. arXiv: 4180v2, 1-22.

[20] Otto, H.H. (1980) The Reciprocal Lattice: An Exercise. Lecture Given at the Universities of Regensburg, TU Berlin and TU Clausthal.

[21] Assmus, E.F. (1985) The American Mathematical Monthly, 92, 213-214. https://doi.org/10.1080/00029890.1985.11971581

[22] Finch, S.R. (2003) Mathematical Constants. In: Encyclopedia of Mathematics and Its Applications, Vol. 94, Cambridge University Press, New York.

[23] Otto, H.H. (2017) Nonlinear Science Letters A, 8, 410-412.

[24] Lange, L.J. (1999) The American Mathematical Monthly, 106, 456-458. https://doi.org/10.1080/00029890.1999.12005070

[25] Mushkolaj, S. (2014) Journal of Modern Physics, 5, 1124-1138. https://doi.org/10.4236/jmp.2014.512115

[26] Olson, S. (2006) The Golden Section: Nature's Greatest Secret. Bloomsbury, New York, $64 \mathrm{p}$.

[27] Sherbon, M.A. (2014) International Journal of Physical Research, 2, 1-9. https://doi.org/10.14419/ijpr.v2i1.1817

[28] Otto, H.H. (2017) Continued Fraction Representations of Universal Numbers and Approximations. Researchgate, 1-4.

[29] Fisher, R.A. (1915) Biometrica, 10, 507-521. https://doi.org/10.2307/2331838

[30] Madelung, E. (1918) Physikalische Zeitschrift, 19, 524-533.

[31] Triebl, R. (2011) Iterative Bestimmung der Madelung-Konstante für zweidimensionale Kristallstrukturen. Physikalische Projektarbeit, TU Graz.

[32] Valleta, M. (2013) Astrophysics and Space Science, 345, 1-9. https://doi.org/10.1007/s10509-013-1388-3

[33] El Naschie, M.S. (2004) Chaos, Solitons \& Fractals, 19, 209-236. https://doi.org/10.1016/S0960-0779(03)00278-9

[34] Cantor, G. (1932) Gesammelte Abhandlungen mathematischen und philosophischen Inhalts. Springer, Berlin. https://doi.org/10.1007/978-3-662-00274-2

[35] El Naschie, M.S. (2017) International Journal of High Energy Physics, 4, 65-74. https://doi.org/10.11648/j.ijhep.20170406.11

[36] Urysohn, P. (1922) Comptes Rendus, 175, 440-442.

[37] Menger, K. (1928) Dimensionstheory. Springer-Verlag, Leipzig und Berlin.

[38] Hausdorff, F. (1918) Mathematische Annalen, 79, 157-179. 
https://doi.org/10.1007/BF01457179

[39] Otto, H.H. (2018) An Exercise: Cauchy Functions Compared to the Gaussian for Diffraction Line Profile Fitting. Researchgate.

[40] Uhlenbeck, G.E. and Goudsmit, S. (1926) Nature, 117, 264-265. https://doi.org/10.1038/117264a0

[41] He, J.H., Tian, D. and Otto, H.H. (2018) Results in Physics, 11, 362-362. https://doi.org/10.1016/j.rinp.2018.09.027

[42] Otto, H.H. (2017) Nonlinear Science Letters A, 8, 413-415.

[43] Odom, B., Hanneke, D., D’Urso, B. and Gabrielse, G. (2006) Physical Review Letters, 97, Article ID: 030801. https://doi.org/10.1103/PhysRevLett.97.030801

[44] He, J.H. (2004) Applied Mathematical Computing, 151, 887-891. https://doi.org/10.1016/S0096-3003(03)00531-9

[45] Lin, L., Yu, D.N., He, C.H. and Liu, Y. (2018) Thermal Science, 22, 1849-1852. https://doi.org/10.2298/TSCI1804849L

[46] Schwinger, J. (1948) Physical Review, 73, 416-417. https://doi.org/10.1103/PhysRev.73.416

[47] Sommerfeld, A. (1919) Atombau und Spektrallinien. Friedrich Vieweg \& Sohn, Braunschweig.

[48] The NIST Reference of Constants, Units and Uncertainty. NIST, Gaithersburg, MD.

[49] Gabrielse, G., Hanneke, D., Kinoshita, T., Nio, M. and Odom, B. (2006) Physical Review Letters, 97, Article ID: 030802. https://doi.org/10.1103/PhysRevLett.97.030802

[50] Gabrielse, G., Hanneke, D., Kinoshita, T., Nio, M. and Odom, B. (2007) Physical Review Letters, 99, Article ID: 039902. https://doi.org/10.1103/PhysRevLett.99.039902

[51] Odom, B. (2004) Fully Quantum Measurement of the Electron Magnetic Momentum. Harvard University, Cambridge, MA.

[52] Otto, H.H. (2018) Does Nature Journal Once again Oversleep the New Era of Physics. Researchgate.

[53] Coldea, R., Tennant, D.A., Wheeler, E.M., Wawrzynska, E., Prabhakaram, D., Telling, M., Habicht, K., Smeibidl, P. and Kiefer, K. (2010) Science, 327, 177-180. https://doi.org/10.1126/science.1180085

[54] Prester, M. (1999) Physical Review B, 60, 3100-3103. https://doi.org/10.1103/PhysRevB.60.3100

[55] Gauthier, R. (2013) Transluminal Energy Quantum Models of the Photon and the Electron. In: The Physics of Realty: Space, Time, Matter, Cosmos, World Scientific, Hackensack, NJ, 445-452. https://doi.org/10.1142/9789814504782_0045

[56] El Naschie, M.S., Olsen, S., Helal, M.A., Marec-Crnjac, L. and Nada, S. (2018) International Journal of Innovation in Science and Mathematics, 6, 11-13.

[57] England. J.L. (2013) Journal of Chemical Physics, 139, Article ID: 121923. https://doi.org/10.1063/1.4818538

[58] Pitkänen, M. (2015) Jeremy England's Vision about Life and Evolution: Comparison with TGD Approach. Researchgate.net, 1-9.

[59] Rafelski, J. and Ericson, T. (2019) The Tale of the Hagedorn Temperature. In: Rafelski, J., Ed., Melting Hadrons, Boiling Quarks-From Hagedorn Temperature to Ultra-Relativistic Heavy-Ion Collisions at CERN, Springer, Cham, 41-48. https://doi.org/10.1007/978-3-319-17545-4_6 
[60] Menger, K. (1928) Dimensionstheorie. B. G. Teubner Publisher, Stuttgart. https://doi.org/10.1007/978-3-663-16056-4

[61] Bohr, N. (1934) Atomic Theory and the Description of Nature. Cambridge University Press, Cambridge, UK.

[62] Bedau, H. and Opperheim, P. (1961) Synthese, 13, 201-232. https://doi.org/10.1007/BF00489884 


\section{Appendix I}

\section{About the Meaning of the Terms Reciprocity, Duality and Complementarity}

These terms, omnipresent in many disciplines of science (physics, mathematics, philosophy, music, economy, etc.) can certainly have different meanings, even if they have something in common.

In mathematics, a reciprocal of a number is its multiplicative inverse, but an inverse is not necessarily a reciprocal. Reciprocity in the amounts of matter and dark matter is formulated according to Equation (39) of the main text. Important for mathematics and physics, a reciprocal vector system can be created by Fourier transformation. However, reciprocity in physics may have a more general meaning when describing a mutual dependence or influence.

Duality in mathematics can be demonstrated on platonic solids, also familiar for a crystallographer. The convex hull of the center points of each face of a starting polyhedron results in a dual polyhedron such that, for instance, the cube and the octahedron form a dual pair, but the tetrahedron is self-dual. In physics, the most prominent example for duality is that between matter and piloting wave in the sense of the De Brogly-Bohm approach [12]. According to the $I R$ theory of Suleiman [8] the relation between matter density and the dark matter surrounding may be quantified as "reciprocal duality", where an amount of matter is transformed into an equal amount of dark matter depending of the recession velocity respectively redshift of a moving body (Figure 6).

Finally, the concept of complementarity in quantum physics has been formulated and coined by Bohr in his Como lecture of 1927, describing the familiar case of reciprocal uncertainty between position and momentum of an electron as conjugate variables [61]. It means that it is hardly possible to know simultaneously with an arbitrary accuracy the outcomes of these variables. Another example of conjugate variables is the magnetic field strength in comparison to the electric one. An elaborated logical analysis of complementarity has been given by Bedau and Oppenheimer [62]. In his late years Bohr was interested in philosophical aspects of complementarity as given in the Yin and Yang conjugate principle of the ancient Tao, and on his gravestone the Taoist symbol is engraved.

In mathematics, a number and the complement to a number add up to a whole of some amount. If one performs the reciprocal of these numbers and renormalize the resulting values, then complement and primal number change their values [4]. In this way one may speak also of reciprocity when dealing with matter density and the dual dark matter density according to Figure 6.

\section{Appendix II}

Another approach for the gyromagnetic factor used the fifth power of $\tilde{\varphi}$ with the value $\tilde{\varphi}^{5}=0.09092922100312$. An approximation is the inverse Lucas number $L_{5}=11$ as combination of two inversely related irrational numbers (see Equations (13) to (15)) 


$$
\left(\varphi^{-5}-\varphi^{5}\right)^{-1}=(11)^{-1}=0.0909090 \cdots
$$

However, physically more convenient is the expression

$$
\frac{2 \pi \cdot v_{K}}{c}=0.0917012 \cdots
$$

where $v_{K}$ is the Klizing speed and $c$ the speed of light. This term keeps no dimension, as required. If we are working with a speed, according to the $I R$ theory the information offset has to be corrected. The speed transforms as

$$
\frac{v}{v_{0}}=1+\beta
$$

combining the length transformation $\frac{l}{l_{0}}=\frac{1+\beta}{1-\beta}$ with the time transformation $\frac{t}{t_{0}}=\frac{1}{1-\beta}$, where $\beta=v / c$ is the recession speed [8]. Surprisingly, an additional $\left(\frac{t}{t_{0}}\right)^{-1}=1-\beta$ term is needed to give more accuracy and the following simple formula

$$
\begin{aligned}
& \sqrt[5]{\frac{2 \pi v_{K}}{c}\left(1+\frac{2 \pi v_{K}}{c}\right)\left(1-\frac{2 \pi v_{K}}{c}\right)}=\sqrt[5]{\frac{2 \pi v_{K}}{c}\left(1-\left(\frac{2 \pi v_{K}}{c}\right)^{2}\right)}=0.61907254 \\
& \text { leading to } \frac{g_{e}}{2}=1.00116100597109 \text { or } g_{e}=2.00232201
\end{aligned}
$$

Remarkably, this value is almost identical to the result of [37], because

$$
2+\frac{\varphi^{6}}{24}=2.002322003
$$

Only now we are allowed to associate the term $\frac{2 \pi v_{K}}{c}$ with Sommerfelds fine-structure constant $\alpha$ [41] applying

$$
\frac{2 \pi v_{K}}{c}=4 \pi \alpha
$$

where $\alpha$ is a measure of the strength of interaction of an electron and a photon in the quantum electrodynamics theory $(Q E D)$. The charge of the electron in $Q E D$ (Lorentz-Heaviside) units has the numerical value of $e=-\sqrt{4 \pi \alpha}$.

The accurate experimental value for the gyromagnetic constant could be attained from Equation (74) using an adapted fine-structure constant of

$$
\alpha^{\prime}=0.007297279955669
$$

$$
\text { respectively } \quad \alpha^{\prime-1}=137.037362698897742
$$

$$
\text { where } \alpha^{\prime}-\alpha=6.43 \times 10^{-7}
$$

Tackling the problem of the not fully adapted accuracy in comparison to the experimental value, one can multiply the term under the fifth root of Equation (74) by a factor of 0.9999902180 or alternatively reduce the Klitzing speed by a 
factor of 0.99999004931863 respectively the charge of the electron by a factor of 0.99999502464694 . This adjustment may result partly from a correction of $g$ as well as $\alpha$ with respect to the $I R$ theory, besides needed radiative corrections. 